\title{
Characterization and clinical significance of biventricular mechanics in patients with systemic lupus erythematosus by $3 T$ cardiovascular magnetic resonance tissue tracking
}

\author{
Huaxia Pu ${ }^{1 \#}$, Beibei Cui ${ }^{2 \#}$, Jing Liu ${ }^{1}$, Wenzhang $\mathrm{He}^{1}$, Xiaoyue Zhou ${ }^{3}$, Hui Lin ${ }^{2}$, Liqing Peng ${ }^{1 \wedge}$ \\ ${ }^{1}$ Department of Radiology, West China Hospital, Sichuan University, Chengdu, China; ${ }^{2}$ Department of Rheumatology and Immunology, West China \\ Hospital, Sichuan University, Chengdu, China; ${ }^{3} \mathrm{MR}$ Collaboration, Siemens Healthineers Ltd., Shanghai, China
}

Contributions: (I) Conception and design: H Pu, B Cui, H Lin, L Peng; (II) Administrative support: H Lin, L Peng, X Zhou; (III) Provision of study materials or patients: H Pu, B Cui, H Lin, L Peng; (IV) Collection and assembly of data: H Pu, B Cui, J Liu, W He; (V) Data analysis and interpretation: $\mathrm{H} \mathrm{Pu}$, J Liu, W He; (VI) Manuscript writing: All authors; (VII) Final approval of manuscript: All authors.

"These authors contributed equally to this work.

Correspondence to: Liqing Peng. Department of Radiology, West China Hospital, Sichuan University, 37 Guoxue Alley, Chengdu 610041, China. Email: pengliqing@wchscu.cn; Hui Lin. Department of Rheumatology and Immunology, West China Hospital, Sichuan University, 37 Guoxue Alley, Chengdu 610041, China. Email: 1hacd@163.com.

Background: Detecting impaired left ventricle (LV) or right ventricle (RV) mechanics could aid in fully understanding the process of cardiac involvement in patients with systemic lupus erythematosus (SLE). This study aimed to evaluate biventricular strain parameters derived from cardiac magnetic resonance (CMR) tissue tracking in SLE patients and their association with other clinical variables.

Methods: A group of 47 SLE patients and 27 healthy controls were enrolled and underwent CMR examination, including cine and late gadolinium enhancement (LGE) imaging. Aside from RV strain parameters in the radial direction, biventricular global peak strain and peak systolic/diastolic global strain rate in radial, circumferential, and longitudinal directions were assessed for each participant. Multivariate linear regression analysis was used to analyze the factors related to the biventricular strain parameters. Receiver operating characteristic (ROC) analysis was used to identify RV dysfunction.

Results: Compared with the controls, part of the biventricular strain parameters in the SLE subgroup with preserved ejection fraction (EF) were impaired, which was more significant in the SLE subgroup with reduced EF (all P<0.05). The SLE patients with RV dysfunction (15/47) included patients with LV dysfunction (8/47). The RVEF was associated with impaired LV global peak strain and peak diastolic strain rate in the SLE patients (absolute value of $\beta=0.406-0.715$, all $\mathrm{P}<0.05)$. The LV LGE in SLE patients $(12 / 47)$ was associated with LV global longitudinal peak strain and peak diastolic global longitudinal strain rate $(\beta=0.378$ and -0.342 ; all $\mathrm{P}<0.05)$. There were independent correlations between pulmonary arterial hypertension and RV global longitudinal peak strain, anti-ribonucleoprotein (RNP) antibody and RV global circumferential peak strain, and pericardial effusion and RV peak diastolic global circumferential strain rate, respectively $(\beta=0.319,0.359$, and -0.285 , respectively; all $\mathrm{P}<0.05)$. The $\mathrm{LV}$ global longitudinal peak strain had greater diagnostic accuracy for RV dysfunction RV dysfunction [area under curve (AUC): 0.933 , cut-off value: $-13.38 \%$ ).

Conclusions: Biventricular strain parameters derived from CMR are sensitive markers of subclinical ventricular function impairment before EF reduction at an early stage of SLE. Biventricular strain analysis could be considered for inclusion in early cardiac functional assessment in SLE patients, particularly LV global longitudinal peak strain, which might assist in therapeutic decision-making and disease monitoring.

\footnotetext{
^ ORCID: Huaxia Pu, 0000-0002-0271-4993; Liqing Peng, 0000-0002-2376-3268.
} 


\begin{abstract}
Keywords: Systemic lupus erythematosus (SLE); cardiovascular magnetic resonance; tissue tracking; myocardial strain; strain rate
\end{abstract}

Submitted May 20, 2021. Accepted for publication Aug 31, 2021.

doi: $10.21037 /$ qims-21-520

View this article at: https://dx.doi.org/10.21037/qims-21-520

\section{Introduction}

Systemic lupus erythematosus (SLE) is an autoimmunemediated, chronic inflammatory connective tissue disease with multi-system involvement. The heart is a major target organ in SLE patients, with a subclinical prevalence reported as high as $80 \%$ in early postmortem studies (1). Cardiovascular involvement in SLE is a complex and dynamic process and can affect any component of the heart, presenting as pericarditis, myocarditis, myocardiopathy, valvular disease, coronary artery disease (CAD), or with conduction abnormalities (2). The underlying pathology includes the deposition of immune complexes in the vascular wall and perivascular tissues, complement system activation, and inflammatory cell infiltration, resulting in diffuse inflammation and myocardial fibrosis (3-5). The cardiac involvement carries an ominous prognosis, manifested as late-stage cardiac dysfunction, heart failure, and increased morbidity and mortality $(6,7)$. However, due to the clinically asymptomatic and non-specific manifestations and lack of reliable examination methods, only $5-10 \%$ of SLE patients usually receive a diagnosis (8). Early detection of cardiac involvement is essential for timely initiation of immunosuppressive treatment that may prevent or reverse cardiac damage progression.

Cardiac magnetic resonance (CMR) imaging is a non-invasive, non-radiating imaging method that can comprehensively assess the heart's structure, function, and tissue characteristics in a one-stop-shop scanning. The CMR tissue tracking technology that tracks features of the images and recognizes them in the contiguous image of the cine sequence can be used to quantitatively evaluate the displacement, motion function, and deformation of the myocardium in global or regional mode, particularly for the left ventricle $(\mathrm{LV})$ and right ventricle $(\mathrm{RV})(9,10)$. Of note, CMR deformation analysis has been shown to detect early abnormalities of the $\mathrm{LV}$ or RV contractile dysfunction beyond conventional global functional assessment with ejection fraction (EF) and provide prognostic information in some cardiovascular diseases (10-13).

To the best of our knowledge, there have been only limited studies $(14,15)$ published examining the feasibility and additive value of biventricular deformation (or mechanics) analysis derived from CMR for cardiac involvement in patients with SLE. Moreover, myocardial strain rate was reported, which could present the rate of myocardial contraction and relaxation, which are more sensitive for identifying myocardial systolic or diastolic functional impairment in some studies $(10,16)$. Our study aimed to evaluate the $\mathrm{LV}$ and RV strain in SLE patients and the relationship of strain indices with other CMRbased [late gadolinium enhancement (LGE); LVEF; RVEF], echocardiography-based [pulmonary arterial hypertension (PAH); pericardial effusion], and laboratory auto-antibody parameters.

We present the following article following the Standards for Reporting Diagnostic accuracy studies (STARD) reporting checklist (available at https://dx.doi.org/10.21037/ qims-21-520).

\section{Methods}

\section{Study design and population}

From 20 February 2017 to 1 December 2019, 53 patients with SLE were consecutively recruited and underwent CMR at our institution. All patients fulfilled at least 4/11 American College of Rheumatology (ACR) revised criteria for SLE classification (17). The disease activity, duration, and medical treatment programs of the SLE patients did not affect enrollment. A total of 27 age- and gender-matched healthy volunteers without cardiac morbidities, relevant medical history, or medications were included as the control group. They underwent the same CMR examination to establish baseline strain values. Exclusion criteria for the 2 groups included clinical evidence of $\mathrm{CAD}$, severe renal failure (estimated glomerular filtration rate $\leq 30 \mathrm{~mL} /$ $\min / 1.73 \mathrm{~m}^{2}$ ), severe claustrophobia, device implantation, allergy to the CMR contrast material, incomplete CMR data, and poor image quality. This study was approved by the Biomedical Research Ethics Committee of our hospital and complied with the mandate of the Declaration 
of Helsinki (2013 edition). All participants gave written informed consent before undergoing imaging.

The clinical manifestations, SLE disease activity index [SLEDAI (18)], auto-antibodies [anti-double-stranded (antidsDNA), anti-Smith (anti-Sm), and anti-ribonucleoprotein (anti-RNP)], and echocardiography findings of the SLE participants were recorded.

\section{CMR scanning protocol}

All participants underwent a standard clinical protocol using 3T magnetic resonance imaging (MRI) scanners (MAGNETOM Trio or MAGNETOM Skyra, Siemens Healthcare, Erlangen, Germany). Data acquisition was performed with a manufacturer's standard electrocardiogram (ECG)-triggering device that monitored each participant's dynamic ECG changes during the end-inspiratory breathhold period. A balanced steady-state free precession (b-SSFP) sequence was used to acquire cine images of the 8 to 12 matching short-axis planes encompassing the entire $\mathrm{LV}$ and $\mathrm{RV}$ and 3 radial long-axis planes (3-, 4-chamber, and LV 2-chamber views). The cardiac frames number of each short-axis plane was 25 . The parameters for cine imaging were as follows: field of view (FOV), $250 \times 300 \mathrm{~mm}^{2}$; matrix size, $208 \times 139$ pixels; iPAT, 2 ; repetition time (TR), $3.3 \mathrm{~ms}$; echo time (TE), $1.22 \mathrm{~ms}$; slice thickness, $8 \mathrm{~mm}$; and flip angle, $40^{\circ}$. The LGE images were acquired by segmented-turbo-FLASH-phase-sensitive inversion recovery (PSIR) sequences (TR, $3.0 \mathrm{~ms}$; TE, $1.18 \mathrm{~ms}$; flip angle, $40^{\circ}$; slice thickness, $8 \mathrm{~mm}$; FOV, $400 \times 270 \mathrm{~mm}^{2}$; and matrix size, $256 \times 148$ pixels) $10-15$ minutes after intravenous injection of gadopentetate dimeglumine (dose: $0.2 \mathrm{~mL} / \mathrm{kg}$ body weight, flow rate: $2.5-3.0 \mathrm{~mL} / \mathrm{s}$, MultiHance $0.5 \mathrm{mmol} / \mathrm{mL}$; Bracco, Milan, Italy).

\section{Imaging analysis}

All the CMR images were analyzed using an offline commercially available software (CVI42, version 5.11.3; Circle Cardiovascular Imaging Inc., Calgary, AB, Canada) based on an incompressible volume-based algorithm (19). For the cine images, the endocardial and epicardial borders of the LV and RV myocardium were manually traced in short-axis slices at the end of diastole and systole in the CVI42 short- three-dimensional (3D) module. Then, global parameters of LV and RV geometry and function, including the end-diastolic volume (EDV), end-systolic volume (ESV), myocardial mass at end-diastole, and
EF, were computed automatically. An LVEF $<50 \%$ and RVEF $<45 \%$ were defined as ventricular dysfunction (20). Biventricular mass, EDV, and ESV were performed using the index to body surface area (BSA). The peak strain and strain rate were obtained by manually delineating the biventricular endocardium and epicardium of the cine images at end-diastole from the short-axis and longaxis 2 - and 4-chamber slice views in the tissue tracking module. The accuracy of tissue tracking for endocardial and epicardial contours was visually checked via automated strain analysis on the CVI42 tissue tracking model, and good quality tracking was obtained in all participants after optimal adjustment. In all series, the papillary muscles and trabeculae were carefully excluded (Figure 1). Strain represents the amplitude of relative thickening, lengthening, and shortening of the myocardium from enddiastole (reference phase), and strain rate represents the rate of myocardial deformation. For a given phase " $n$ ", the formula was calculated as: strain $=\left(\mathrm{L}_{\mathrm{n}+1}-\mathrm{L}_{\mathrm{n}}\right) / \mathrm{L}_{\mathrm{n}}$, which was expressed as $\%$. Strain rate $=\left(\right.$ strain $_{n+1}-$ strain $\left._{n}\right)$ temporal resolution, which was expressed as $\mathrm{s}^{-1}(16)$. The positive and negative signs of the measurement values indicate the direction of myocardial deformation. The deformation parameters calculated automatically by the software included 3D global radial peak strain (GRS), global circumferential peak strain (GCS), global longitudinal peak strain (GLS), peak systolic/diastolic global radial strain rate (PS/PD-GRSR), peak systolic/diastolic global circumferential strain rate (PS/PD-GCSR), and peak systolic/diastolic global longitudinal strain rate (PS/PDGLSR) of the LV and RV. As observed in Claus et al. (10), $\mathrm{RV}$ radial strain measurements displayed low repeatability and high variability due to the complex morphological structure and relatively thin ventricular wall; related parameters were not applied. The curves graphs of the strain variables showed the global peak strain changes over time in a cardiac cycle (Figure 2). In addition, LGE was confirmed by its presence in 2 perpendicular views, with the consensus of 2 radiologists. When LGE was identified, its pattern (e.g., linear, patchy, and diffuse), location (e.g., interventricular septum, LV free wall, and pericardium), and distribution (e.g., intramyocardial, subendocardial, transmural, and subepicardial) were recorded.

\section{Reproducibility}

A total of 25 participants (15 SLE patients and 10 controls) were randomly selected to assess the reproducibility and 

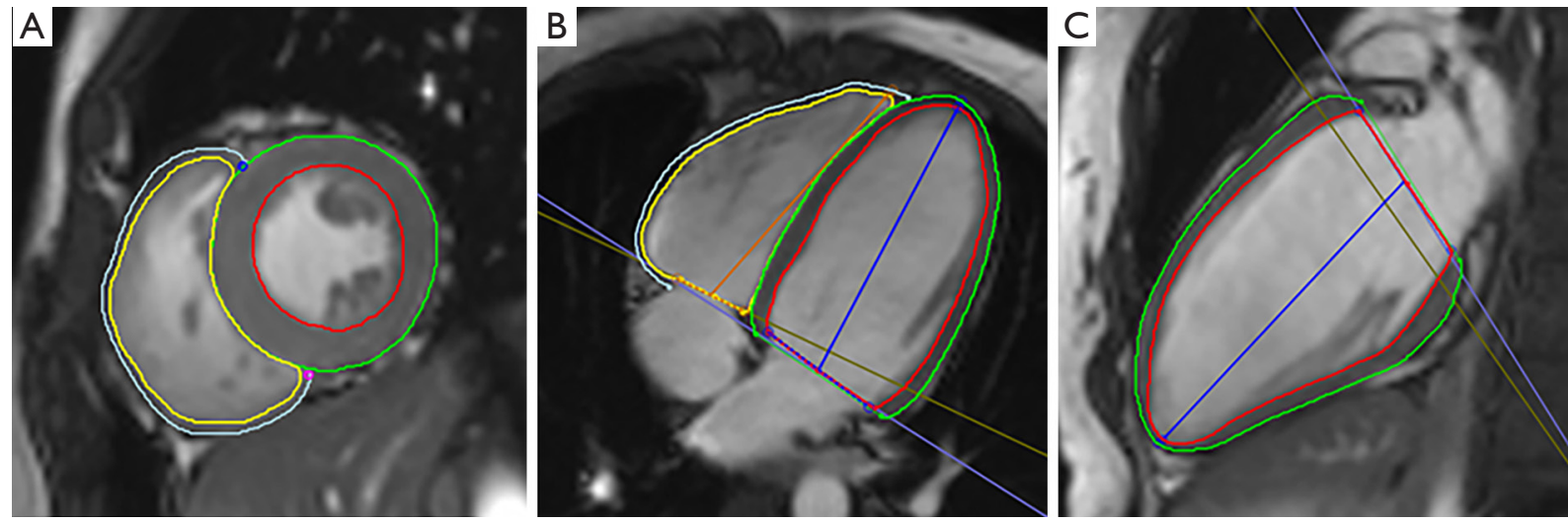

Figure 1 The operation of biventricular strain evaluation in post-processing software. Images show the delineated endocardial and epicardial borders of ventricles at the end-diastolic phase of short-axis (A), 2-chamber long-axis and 4-chamber long-axis cine images (B,C). Red and green curves show left ventricle endocardial and epicardial contours, respectively. Yellow and light blue curves show right ventricle endocardial and epicardial contours, respectively.
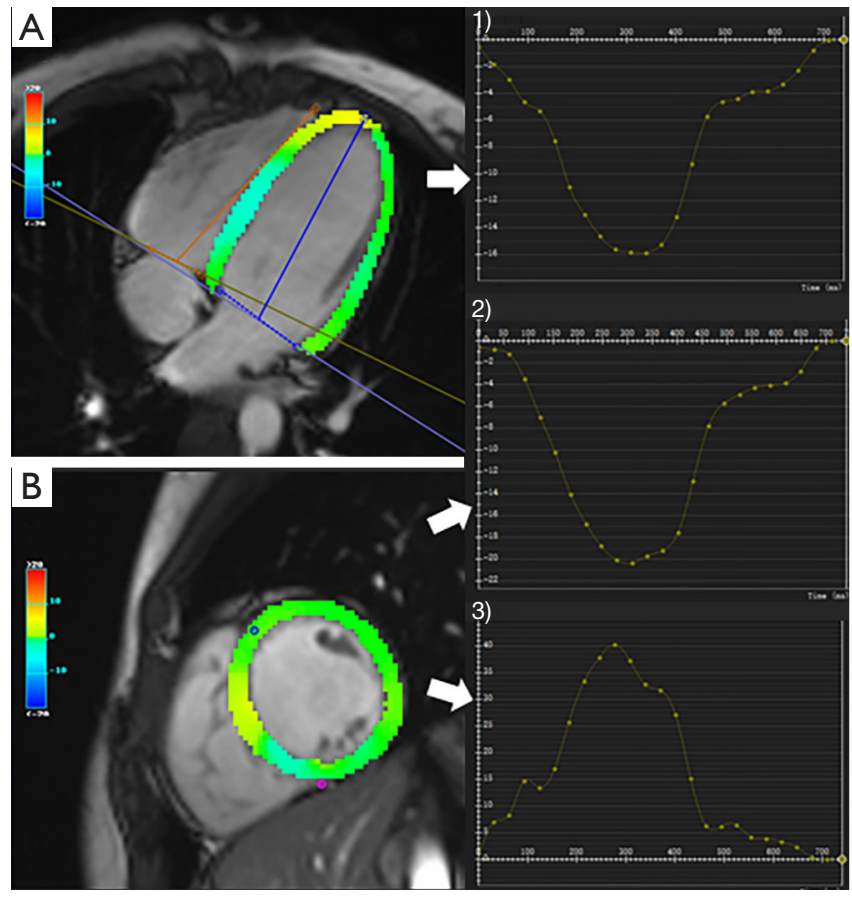
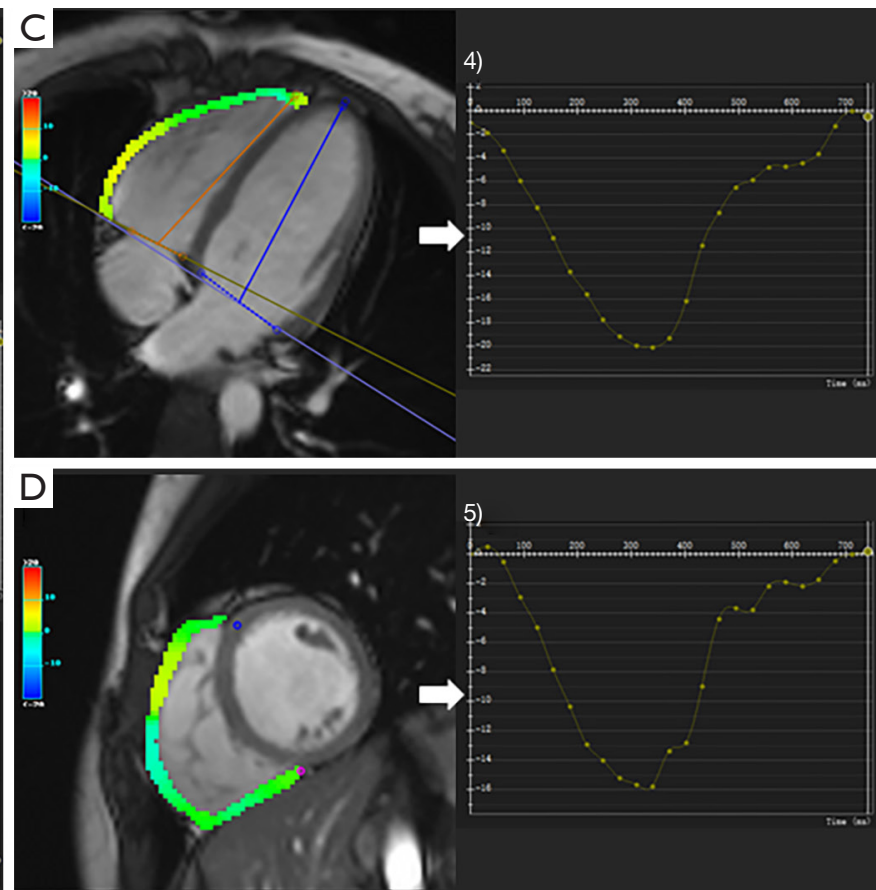

Figure 2 The color-coded diagrams and curves of biventricular global peak strain parameters derived from CMR tissue tracking. (A,B) and (C,D) are color-coded diagrams of the LV and RV global peak strain values (take radial strain for example), respectively. From the LV long-axis 4 chamber SSFP cine image (A), the longitudinal strain curve is derived [1], and the short-axis cine image (B) is used to calculate the circumferential [2] and radial strain curves [3]. RV 4-chamber SSFP cine image (C) allows for the calculation of the longitudinal strain curve [4], and the short-axis cine image (D) is used to derive the circumferential [5] strain curve. CMR, cardiac magnetic resonance; LV, left ventricular; RV, right ventricular; SSFP, steady-state free precession. 


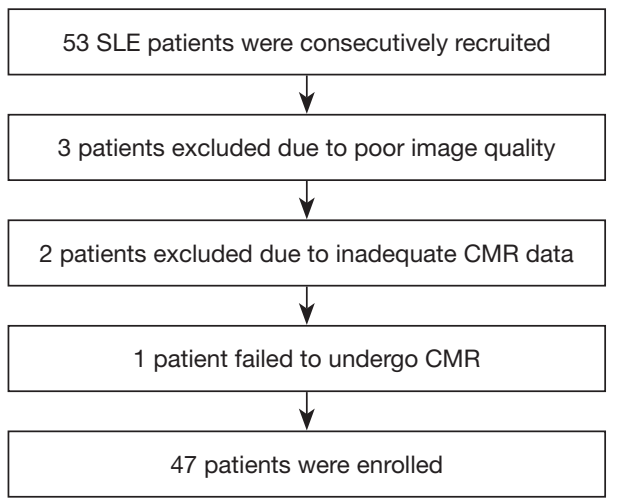

Figure 3 Flow diagram of SLE patients' enrollment. SLE, systemic lupus erythematosus; CMR, cardiac magnetic resonance.

reliability of biventricular myocardial strain measurement derived from CMR tissue tracking. To determine intraobserver variability, 1 observer evaluated the same participants on 2 separate measurements 1-month apart. For the interobserver variability evaluation, another experienced investigator reanalyzed the parameters while blinded to the results produced by the first observer. The mean values of the 2 observers were taken as the final results for further analysis.

\section{Statistical analysis}

Continuous variables were expressed as mean \pm standard deviation (SD), and categorical data as percentages. The statistical analysis was performed using SPSS statistical software version 23 for Windows (IBM Corp., Armonk, NY, USA) and GraphPad Prism (version 7.0c; GraphPad Software, San Diego, CA, USA). The KolmogorovSmirnov test was used to examine the distribution and normality of the data. Homogeneity of variance was evaluated using Levene's test. The independent sample t-test or Mann-Whitney $U$ test was used to compare parameters between different groups. Strain parameters among normal controls and SLE subgroups based on EF were compared by one-way repeated analysis of variance (ANOVA) or the Kruskal-Wallis rank test. The Student-Newman-Keuls (SNK) or least-significant difference (LSD) test was used for multiple pairwise comparisons when the $\mathrm{P}$ value of oneway ANOVA was less than 0.05 . Association between the statistically significant CMR-based and clinic parameters obtained by the above subgroup analysis and biventricular strain parameters were further assessed using univariate and backward stepwise multivariate linear regression analyses.
Pearson's or Spearman's correlation coefficients were used to analyzing ventricular global peak strain, diastolic strain rate, and corresponding ventricular EF. The strength of the correlation was described by the absolute value of $\mathrm{r}$ (0.8-1.0: very strong, $0.8-0.6$ : moderately strong, $0.6-0.3$ : fair, and $<0.3$ : weak) (21). The sensitivity and specificity of biventricular global peak strain cut-off points were decided by the receiver operating characteristic (ROC) curve to identify RV dysfunction (RVEF $<45 \%$ ) in the SLE patients. The reliability and reproducibility of parameters for interand intra-observer variability were evaluated by intraclass correlation coefficient (ICC) $(\geq 0.75$ : excellent, $<0.75$ and $\geq 0.40$ : moderate, and $<0.40$ : poor). A 2 -tailed $\mathrm{P}$ value $<0.05$ was considered statistically significant.

\section{Results}

\section{Participant characteristics}

A total of 47 patients with SLE (mean age, 37.74 11.58 years; male:female $=3: 44$ ) and 27 age- and gendermatched healthy controls (mean age, $41.44 \pm 12.13$ years; male:female $=4: 23$ ) were enrolled in our study (Figure 3). All the baseline characteristics for the study population are summarized in Table 1. Compared to the control participants, the LVEF and RVEF were significantly reduced in participants with SLE, whereas the LV mass index, LV ESV, and RV ESV index were all significantly increased (all $\mathrm{P}<0.05$ ). The LGE was observed in 12 of the 47 SLE participants and was primarily a non-ischemic pattern, including 9 participants with mid-wall LGE in the basal- and mid-septum or LV free wall, 8 participants with linear or patchy enhancement in RV insertion points, 1 participant with transmural LGE, and 1 participant with pericardial enhancement (Figure 4).

\section{Biventricular strain analysis}

To evaluate the association between conventional global cardiac function (assessed by EF) and strain parameters, SLE participants were classified into reduced EF and preserved EF subgroups based on LVEF $<50 \%$ and RVEF $<45 \%$ (20). The strain parameters based on CMR tissue tracking of all participants are shown in Table 2. Those with SLE showed significantly lower LV GRS, GLS, PD-GRSR, RV GCS, GLS, and PDGLSR than the normal controls (all $\mathrm{P}<0.05$ ). For subgroups of SLE patients, the subgroup with preserved LVEF showed significantly lower LV GLS, 
Table 1 Baseline characteristics

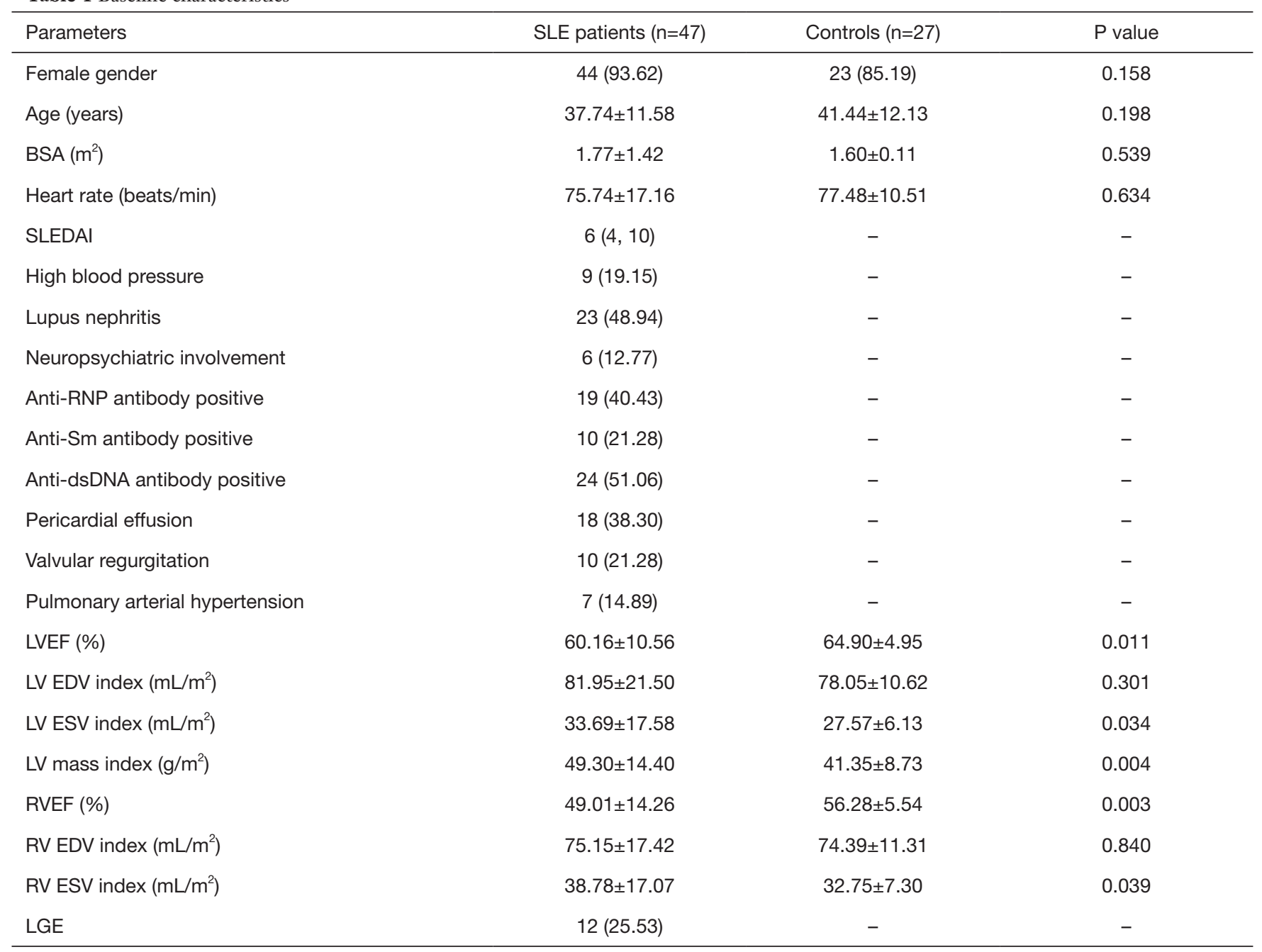

Values are presented as the mean $\pm \mathrm{SD}, \mathrm{n}(\%)$, or median (interquartile). BSA, body surface area; SLEDAl, systemic lupus erythematosus disease activity index; anti-dsDNA, anti-double-stranded DNA; anti-Sm, anti-Sm; anti-RNP, anti-ribonucleoprotein; LV, left ventricle; EF, ejection fraction; EDV, end diastolic volume; ESV, end systolic volume; RV, right ventricle; LGE, late gadolinium enhancement.

RV GCS, and PDGLSR than the normal controls, whereas the subgroup with reduced LVEF had lower LV strain, PSGRSR, PS-GCSR, PD strain rate, RV GCS, GLS, and PDGCSR than the preserved LVEF group (all $\mathrm{P}<0.05$ ) significantly. The preserved RVEF group showed lower RV GCS and PDGLSR than the normal controls, whereas the reduced RVEF group had significantly lower LV strain, PD strain rate, RV GCS, GLS, and PDGCSR than the preserved RVEF subgroup (all $\mathrm{P}<0.05$ ). Furthermore, compared with the normal group, except that the reduced LVEF group had a lower LV PS strain rate in all directions, and the reduced RVEF group had a lower LV PSGLSR and RV PSGCSR, the reduced LVEF/RVEF group presented with a significantly lower LV strain and PD strain rate in all directions and an RV strain and PD strain rate in the circumferential and longitudinal directions (all $\mathrm{P}<0.05$ ).

In addition, based on the presence of LGE, PAH, and pericardial effusion, the SLE patients were divided into 2 groups, namely, positive and negative subgroups. For the immunological indicators (anti-RNP, anti-Sm, and antidsDNA antibodies), the SLE participants were classified into 2 groups based on positive antibodies. Comparisons between the strain parameters in the SLE patients after grouping are shown in Tables 3,4. The presence of LGE was associated with LV GLS and PDGLSR ( $\mathrm{P}=0.043$ and 0.019 , respectively). The presence of $\mathrm{PAH}$ was associated with $\mathrm{RV}$ 

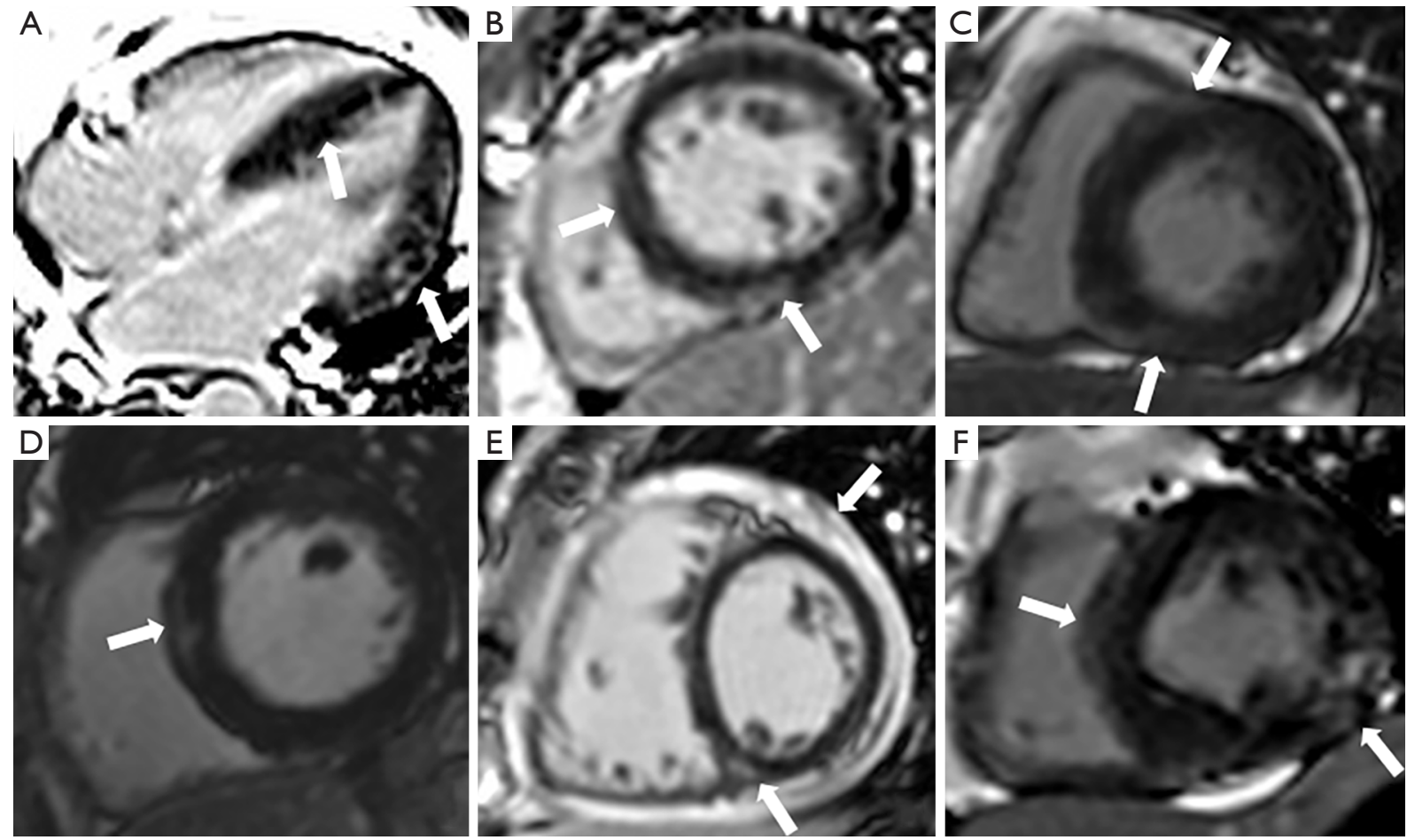

Figure 4 Representative images of LGE in patients with systemic lupus erythematosus. The white arrows indicate the LGE areas of myocardium. (A,B) Patchy LGE in the interventricular septum and LV free wall; (C) LGE in the mid-wall of interventricular septum; (D) LGE in the interventricular septum and RV insertion point; (E) LGE in RV insertion points; (F) LGE in RV insertion point and pericardium. LGE, late gadolinium enhancement; SLE, systemic lupus erythematosus; RV, right ventricular; LV, left ventricular.

GLS $(\mathrm{P}=0.008)$, and the presence of pericardial effusion was associated with RV PDGCSR ( $\mathrm{P}=0.041)$. The presence of anti-RNP antibodies was associated with RV GCS and GLS $(\mathrm{P}=0.006$ and 0.031 , respectively). In addition, there were no associations between biventricular strain parameters and the presence of anti-Sm and anti-dsDNA antibodies $(\mathrm{P}>0.05$ for all).

\section{Univariate and multivariate linear regression analysis}

As shown in Table 5, univariate and backward step multivariate linear regression analyses demonstrated that LVEF was associated with RV GCS, GLS, and RV PDGCSR (all $\mathrm{P}<0.05$ ), but only RV PDGCSR had an independent correlation $(\beta=0.371, \mathrm{P}=0.008$, model $\left.\mathrm{R}^{2}=0.227\right)$. The RVEF was independently associated with $\mathrm{LV}$ strain and strain rate in all directions (absolute value of $\beta=0.406-0.715$, all $P<0.05)$. The LV LGE was associated with LV GLS and PDGLSR $(\beta=0.378,-0.342$; all $\mathrm{P}<0.05)$, and the latter had an independent correlation $(\beta=-0.278$, $\mathrm{P}=0.031$, model $\mathrm{R}^{2}=0.325$ ).
For the clinical indicators, $\mathrm{PAH}$ showed an independent association with RV GLS $(\beta=0.319, \mathrm{P}=0.027$, model $\left.\mathrm{R}^{2}=0.200\right)$. Pericardial effusion showed an association with RV PDGCSR ( $\beta=-0.285, P=0.037$, model $R^{2}=0.227$ ), and anti-RNP antibody showed an independent association with RV GCS ( $\beta=0.359, \mathrm{P}=0.011$, model $\left.\mathrm{R}^{2}=0.215\right)$.

\section{Relationship between EF and strain parameters}

In all directions, $L V$ peak strain had a fair to strong correlation with LVEF (absolute value of $\mathrm{r}=0.336-0.829$, all $\mathrm{P}<0.05$ ), and the correlations were more significant in the reduced EF group (Figure 5). Except for the RV GLS in the SLE patients with preserved RVEF $(\mathrm{P}>0.05)$, RV peak strain in the circumferential and longitudinal directions had a fair to moderately strong correlation with RVEF (absolute value of $\mathrm{r}=0.356-0.572$, all $\mathrm{P}<0.05$ ) (Figure 6). Except for the LV GRSR and GCSR in the SLE patients with preserved LVEF, which were fairly correlated with LVEF (absolute value of $\mathrm{r}=0.356-0.378$, all $\mathrm{P}<0.05)$, the strain rate in the $\mathrm{LV}$ and $\mathrm{RV}$ was not correlated with $\mathrm{EF}$ (all $\mathrm{P}>0.05$ ) (Figures 5,6). 
Table 2 Comparison of LV and RV strain parameters between SLE patients and controls

\begin{tabular}{|c|c|c|c|c|c|c|}
\hline Parameters & $\begin{array}{l}\text { Control participants } \\
\qquad(n=27)\end{array}$ & $\begin{array}{c}\text { SLE participants } \\
(\mathrm{n}=47)\end{array}$ & \multicolumn{4}{|c|}{ SLE patients } \\
\hline \multicolumn{7}{|l|}{ LV } \\
\hline GRS (\%) & $36.96 \pm 4.67$ & $33.07 \pm 10.82^{*}$ & $35.05 \pm 7.75$ & $18.87 \pm 7.64^{\dagger \ddagger}$ & $35.02 \pm 7.69$ & $26.48 \pm 11.49^{\dagger \ddagger}$ \\
\hline GCS (\%) & $-21.79 \pm 1.61$ & $-19.79 \pm 8.11$ & $-22.08 \pm 2.58$ & $-14.52 \pm 3.43^{\dagger \ddagger}$ & $-22.05 \pm 2.37$ & $-18.11 \pm 5.22^{\dagger \ddagger}$ \\
\hline PSGRSR $\left(s^{-1}\right)$ & $1.99 \pm 0.31$ & $1.75 \pm 1.17$ & $1.97 \pm 0.58$ & $1.49 \pm 0.88^{\dagger \neq}$ & $1.98 \pm 0.63$ & $1.69 \pm 0.68$ \\
\hline $\operatorname{PSGCSR}\left(\mathrm{s}^{-1}\right)$ & $-1.12 \pm 0.14$ & $-1.03 \pm 0.50$ & $-1.13 \pm 0.20$ & $-0.90 \pm 0.30^{\dagger \ddagger}$ & $-1.12 \pm 0.18$ & $-1.04 \pm 0.32$ \\
\hline PSGLSR $\left(\mathrm{s}^{-1}\right)$ & $-0.88 \pm 0.20$ & $-0.82 \pm 0.27$ & $-0.84 \pm 0.22$ & $-0.66 \pm 0.39^{\dagger}$ & $-0.85 \pm 0.22$ & $-0.72 \pm 0.33^{\dagger}$ \\
\hline $\operatorname{PDGRSR}\left(\mathrm{s}^{-1}\right)$ & $-2.79 \pm 0.66$ & $-2.34 \pm 0.89^{\star}$ & $-2.55 \pm 0.74$ & $-1.45 \pm 0.81^{\dagger \ddagger}$ & $-2.6 \pm 0.75$ & $-1.84 \pm 0.85^{\dagger \ddagger}$ \\
\hline \multicolumn{7}{|l|}{$\mathrm{RV}$} \\
\hline GCS (\%) & $-14.78 \pm 2.54$ & $-10.39 \pm 7.99^{\star}$ & $-11.93 \pm 4.7^{\dagger}$ & $-7.91 \pm 3.55^{\dagger \ddagger}$ & $-12.54 \pm 4.64^{\dagger}$ & $-8.47 \pm 3.75^{\dagger \ddagger}$ \\
\hline GLS (\%) & $-15.94 \pm 2.45$ & $-14.34 \pm 3.78^{\star}$ & $-15.05 \pm 3.36$ & $-11.95 \pm 4.05^{\dagger \ddagger}$ & $-15.34 \pm 3.35$ & $-12.78 \pm 3.73^{\dagger \neq}$ \\
\hline $\operatorname{PSGCSR}\left(\mathrm{s}^{-1}\right)$ & $-0.85 \pm 0.21$ & $-0.69 \pm 0.46$ & $-0.76 \pm 0.24$ & $-0.71 \pm 0.23$ & $-0.78 \pm 0.24$ & $-0.68 \pm 0.22^{\dagger}$ \\
\hline PSGLSR $\left(s^{-1}\right)$ & $-0.91 \pm 0.20$ & $-0.85 \pm 0.21$ & $-0.86 \pm 0.21$ & $-0.84 \pm 0.21$ & $-0.87 \pm 0.22$ & $-0.85 \pm 0.19$ \\
\hline $\operatorname{PDGCSR}\left(\mathrm{s}^{-1}\right)$ & $0.95 \pm 0.24$ & $0.77 \pm 0.53$ & $0.87 \pm 0.28$ & $0.61 \pm 0.24^{\dagger \ddagger}$ & $0.88 \pm 0.27$ & $0.70 \pm 0.28^{\dagger \ddagger}$ \\
\hline PDGLSR $\left(s^{-1}\right)$ & $1.06 \pm 0.20$ & $0.90 \pm 0.26^{\star}$ & $0.94 \pm 0.24^{\dagger}$ & $0.82 \pm 0.25^{\dagger}$ & $0.92 \pm 0.25^{\dagger}$ & $0.91 \pm 0.24^{\dagger}$ \\
\hline
\end{tabular}

Values are presented as the mean \pm SD. SLE, systemic lupus erythematosus; LV, left ventricle; RV, right ventricle; EF, ejection fraction; GRS, global radial peak strain; GCS, global circumferential peak strain; GLS, global longitudinal peak strain; PSGRSR, peak systolic GRS rate; PSGCSR, peak systolic GCS rate; PSGLSR, peak systolic GLS rate; PDGRSR, peak diastolic GRS rate; PDGCSR, peak diastolic GCS rate; PDGLSR, peak diastolic GLS rate. ${ }^{*} \mathrm{P}<0.05$, SLE patients versus control subjects. ${ }^{\dagger} \mathrm{P}<0.05$, versus control subjects; ${ }^{\ddagger} \mathrm{P}<0.05$, vs. patients with preserved LVEF/RVEF group.

\section{ROC curve analysis of biventricular global peak strain to predict $R V$ dysfunction}

The ROC curve analysis showed that when LV GRS $<28.49 \%$ [area under the curve (AUC): 0.742, sensitivity: 66.7\%, specificity: $84.38 \%$ ), LV GCS >-19.86\% (AUC: 0.769 , sensitivity: $73.3 \%$, specificity: $84.38 \%)$, LV GLS >-13.38\% (AUC: 0.933 , sensitivity: $86.7 \%$, specificity: 84.38\%), RV GCS >-11.79\% (AUC: 0.744, sensitivity: 80.0\%, specificity: $65.63 \%$ ), and RV GLS >-14.54\% (AUC: 0.700, sensitivity: $73.3 \%$, specificity: $65.63 \%$ ), it had a diagnostic potential for RV dysfunction (RVEF <45\%) in SLE patients (Figure 7).

\section{Intra-observer and inter-observer variability}

As demonstrated in Table 6, there were moderate-to- excellent intra- and inter-observer agreements in the measurement of biventricular global peak strain (ICC $=0.875$ to 0.957 and 0.832 to 0.939 , respectively) and biventricular strain rate (ICC $=0.622$ to 0.890 and 0.627 to 0.857 , respectively).

\section{Discussion}

Tissue tracking with CMR has been increasingly used to quantitatively measure $\mathrm{LV}$ or RV strain parameters from the radial, circumferential, and longitudinal directions to assess ventricular function with excellent sensitivity and reproducibility $(12,22-25)$. To our knowledge, this is the first study involving a comprehensive evaluation of biventricular strain and strain rate in SLE patients and their relationship with EF, LGE, PAH, pericardial effusion, and 
Table 3 Comparison of LV and RV strain parameters between SLE subgroups based on LGE, PAH, and pericardial effusion

\begin{tabular}{|c|c|c|c|c|c|c|c|c|c|}
\hline Parameters & $\mathrm{LGE}+(\mathrm{n}=12)$ & LGE- $(n=35)$ & $P$ value & $\mathrm{PAH}(\mathrm{n}=7)$ & $\begin{array}{c}\text { Non-PAH } \\
(n=40)\end{array}$ & $P$ value & $\begin{array}{c}\text { Pericardial } \\
\text { effusion+ }(n=18)\end{array}$ & $\begin{array}{c}\text { Pericardial } \\
\text { effusion- }(n=29)\end{array}$ & $P$ value \\
\hline \multicolumn{10}{|l|}{ LV } \\
\hline GCS (\%) & $-19.07 \pm 4.57$ & $-21.38 \pm 3.59$ & 0.080 & $-18.67 \pm 6.24$ & $-20.99 \pm 3.53$ & 0.338 & $-21.00 \pm 4.51$ & $-20.67 \pm 3.63$ & 0.785 \\
\hline $\operatorname{PSGCSR}\left(\mathrm{s}^{-1}\right)$ & $-1.05 \pm 0.29$ & $-1.11 \pm 0.21$ & 0.468 & $-1.07 \pm 0.34$ & $-1.09 \pm 0.22$ & 0.789 & $-1.13 \pm 0.25$ & $-1.07 \pm 0.22$ & 0.458 \\
\hline $\operatorname{PSGLSR}\left(\mathrm{s}^{-1}\right)$ & $-0.68 \pm 0.24$ & $-0.85 \pm 0.26$ & 0.060 & $-0.85 \pm 0.36$ & $-0.79 \pm 0.25$ & 0.633 & $-0.83 \pm 0.31$ & $-0.79 \pm 0.23$ & 0.560 \\
\hline PDGRSR $\left(s^{-1}\right)$ & $-2.02 \pm 0.82$ & $-2.48 \pm 0.84$ & 0.106 & $-2.42 \pm 1.34$ & $-2.31 \pm 0.80$ & 0.890 & $-2.54 \pm 0.96$ & $-2.25 \pm 0.78$ & 0.273 \\
\hline GCS (\%) & $-11.16 \pm 4.54$ & $-11.27 \pm 4.87$ & 0.945 & $-9.17 \pm 5.31$ & $-11.61 \pm 4.55$ & 0.213 & $-10.45 \pm 5.17$ & $-11.73 \pm 4.48$ & 0.374 \\
\hline GLS (\%) & $-14.98 \pm 3.84$ & $-14.37 \pm 3.61$ & 0.618 & $-11.23 \pm 3.59$ & $-15.07 \pm 3.33$ & 0.008 & $-13.33 \pm 3.91$ & $-15.27 \pm 3.31$ & 0.075 \\
\hline PSGCSR $\left(s^{-1}\right)$ & $-0.78 \pm 0.26$ & $-0.74 \pm 0.23$ & 0.656 & $-0.72 \pm 0.12$ & $-0.76 \pm 0.25$ & 0.711 & $-0.70 \pm 0.19$ & $-0.78 \pm 0.26$ & 0.317 \\
\hline PSGLSR $\left(\mathrm{s}^{-1}\right)$ & $-0.87 \pm 0.25$ & $-0.86 \pm 0.19$ & 0.889 & $-0.73 \pm 0.18$ & $-0.89 \pm 0.20$ & 0.076 & $-0.82 \pm 0.21$ & $-0.89 \pm 0.20$ & 0.277 \\
\hline $\operatorname{PDGCSR}\left(\mathrm{s}^{-1}\right)$ & $0.83 \pm 0.27$ & $0.82 \pm 0.29$ & 0.925 & $0.74 \pm 0.24$ & $0.85 \pm 0.29$ & 0.374 & $0.72 \pm 0.23$ & $0.89 \pm 0.30$ & 0.041 \\
\hline PDGLSR $\left(s^{-1}\right)$ & $0.98 \pm 0.28$ & $0.89 \pm 0.23$ & 0.312 & $0.91 \pm 0.23$ & $0.92 \pm 0.25$ & 0.960 & $0.86 \pm 0.20$ & $0.95 \pm 0.26$ & 0.218 \\
\hline
\end{tabular}

Values are presented as the mean $\pm \mathrm{SD}$. + means positive, and - means negative. LGE, late gadolinium enhancement; PAH, pulmonary arterial hypertension; LV, left ventricle; RV, right ventricle; GRS, global radial peak strain; GCS, global circumferential peak strain; GLS, global longitudinal peak strain; PSGRSR, peak systolic GRS rate; PSGCSR, peak systolic GCS rate; PSGLSR, peak systolic GLS rate; PDGRSR, peak diastolic GRS rate; PDGCSR, peak diastolic GCS rate; PDGLSR, peak diastolic GLS rate.

laboratory autoimmune antibodies.

The primary findings of our study were as follows: (I) compared with the controls, part of biventricular strain parameters in the SLE subgroup with preserved EF were impaired, which was more significant in the SLE subgroup with reduced EF; the impairment in the biventricular strain rate in the SLE patients comprised primarily the peak diastolic strain rate; (II) the presence of LV LGE was associated with LV GLS and PDGLSR; (III) there were significant interactions in biventricular myocardial function; RV dysfunction occurred before the LV dysfunction; (IV) There were independent correlations between PAH and RV GLS, anti-RNP antibody and RV GCS, and pericardial effusion and RV PDGCSR, respectively; (V) the ROC curve analysis of biventricular peak strain demonstrated that LV GLS had the greatest diagnostic potential for RV dysfunction in SLE patients (AUC: 0.933, cut-off value:
$-13.38 \%)$

Our results showed that biventricular strain parameters were more effective than EF in the early detection of myocardial functional changes in patients with SLE. The EF of the left or right ventricles was generally correlated with the global peak strain of the corresponding ventricle, and the correlation was more significant as the EF was lower than the normal range. Our study also found that the SLE subgroup with preserved LVEF had a significantly impaired LV GLS than normal controls. It was previously proposed that LV myocardial strain derived from speckletracking echocardiography could more sensitively detect $\mathrm{LV}$ systolic dysfunction than LVEF and LV volume, particularly the LV GLS, which could be a marker of subclinical LV dysfunction $(26,27)$. Therefore, CMR tissue tracking technology could be considered in early SLE to detect myocardial function abnormalities so as not to miss these 
Table 4 Comparison of LV and RV strain parameters between SLE subgroups based on auto-antibodies indicators

\begin{tabular}{|c|c|c|c|c|c|c|c|c|c|}
\hline Parameters & $\begin{array}{c}\text { Anti-dsDNA + } \\
(n=24)\end{array}$ & $\begin{array}{l}\text { Anti-dsDNA - } \\
\quad(n=23)\end{array}$ & P-value & $\begin{array}{c}\text { Anti-Sm + } \\
(n=10)\end{array}$ & $\begin{array}{c}\text { Anti-Sm - } \\
(\mathrm{n}=37)\end{array}$ & $P$ value & $\begin{array}{c}\text { Anti-RNP + } \\
(n=19)\end{array}$ & $\begin{array}{c}\text { Anti-RNP - } \\
\quad(n=28)\end{array}$ & $P$ value \\
\hline \multicolumn{10}{|l|}{ LV } \\
\hline GCS (\%) & $-21.17 \pm 3.42$ & $-20.40 \pm 4.47$ & 0.513 & $-20.88 \pm 5.79$ & $-20.77 \pm 3.39$ & 0.939 & $-20.62 \pm 4.94$ & $-20.91 \pm 3.19$ & 0.808 \\
\hline $\operatorname{PSGCSR}\left(\mathrm{s}^{-1}\right)$ & $-1.11 \pm 0.23$ & $-1.08 \pm 0.24$ & 0.664 & $-1.09 \pm 0.33$ & $-1.09 \pm 0.21$ & 0.969 & $-1.15 \pm 0.27$ & $-1.06 \pm 0.20$ & 0.197 \\
\hline PSGLSR $\left(\mathrm{s}^{-1}\right)$ & $-0.83 \pm 0.25$ & $-0.78 \pm 0.28$ & 0.589 & $-0.79 \pm 0.25$ & $-0.81 \pm 0.27$ & 0.854 & $-0.85 \pm 0.30$ & $-0.77 \pm 0.23$ & 0.323 \\
\hline PDGRSR $\left(\mathrm{s}^{-1}\right)$ & $-2.37 \pm 0.75$ & $-2.36 \pm 0.97$ & 0.968 & $-2.28 \pm 0.73$ & $-2.38 \pm 0.89$ & 0.729 & $-2.30 \pm 0.75$ & $-2.40 \pm 0.93$ & 0.709 \\
\hline GCS (\%) & $-11.86 \pm 4.94$ & $-10.60 \pm 4.54$ & 0.365 & $-10.31 \pm 4.48$ & $-11.50 \pm 4.84$ & 0.490 & $-9.00 \pm 4.07$ & $-12.77 \pm 4.61$ & 0.006 \\
\hline GLS (\%) & $-14.29 \pm 3.36$ & $-14.77 \pm 3.97$ & 0.653 & $-13.42 \pm 4.15$ & $-14.82 \pm 3.49$ & 0.284 & $-13.15 \pm 3.97$ & $-15.46 \pm 3.13$ & 0.031 \\
\hline $\operatorname{PSGCSR}\left(\mathrm{s}^{-1}\right)$ & $-0.78 \pm 0.24$ & $-0.71 \pm 0.23$ & 0.330 & $-0.70 \pm 0.21$ & $-0.76 \pm 0.24$ & 0.429 & $-0.67 \pm 0.20$ & $-0.80 \pm 0.25$ & 0.080 \\
\hline PSGLSR $\left(\mathrm{s}^{-1}\right)$ & $-0.83 \pm 0.14$ & $-0.89 \pm 0.26$ & 0.367 & $-0.79 \pm 0.17$ & $-0.88 \pm 0.21$ & 0.209 & $-0.82 \pm 0.18$ & $-0.89 \pm 0.22$ & 0.322 \\
\hline PDGCSR $\left(s^{-1}\right)$ & $0.85 \pm 0.29$ & $0.80 \pm 0.28$ & 0.529 & $0.88 \pm 0.24$ & $0.81 \pm 0.3$ & 0.519 & $0.74 \pm 0.25$ & $0.89 \pm 0.29$ & 0.078 \\
\hline PDGLSR $\left(\mathrm{s}^{-1}\right)$ & $0.91 \pm 0.24$ & $0.92 \pm 0.25$ & 0.936 & $0.88 \pm 0.21$ & $0.93 \pm 0.25$ & 0.565 & $0.94 \pm 0.20$ & $0.90 \pm 0.27$ & 0.522 \\
\hline
\end{tabular}

Values are presented as the mean \pm SD. + means positive, and - means negative. Anti-dsDNA, anti-double-stranded DNA; anti-Sm, antiSmith; anti-RNP, antiribonucleoprotein; GRS, global radial peak strain; GCS, global circumferential peak strain; GLS, global longitudinal peak strain; PSGRSR, peak systolic GRS rate; PSGCSR, peak systolic GCS rate; PSGLSR, peak systolic GLS rate; PDGRSR, peak diastolic GRS rate; PDGCSR, peak diastolic GCS rate; PDGLSR, peak diastolic GLS rate.

during EF evaluation. LVEF reduction in different cardiac diseases has been widely discussed and confirmed to be associated with poor prognosis. Thus, if CMR results reveal myocardial strain impairment, clinicians might consider implementing early anti-ventricular remodeling treatment to improve patient prognosis.

In our study, there were global peak strain changes in the SLE patients whether the LVEF or RVEF was preserved or not. Regarding the global peak strain rate, SLE patients primarily demonstrated the impairment of peak diastolic strain rate compared with the control group. This was also observed in the comparison between the EF reduction subgroup and EF preservation subgroup. According to the literature, early or subclinical diastolic dysfunction is common in SLE patients with normal LVEF using 2D echocardiography or the stain rate of tissue Doppler $(28,29)$.

We also found that LGE positivity in the LV wall was associated with LV GLS and PDGLSR, and the latter had an independent correlation. du Toit et al. (30) recently found that the LV GLS based on speckle-tracking echocardiography may be an independent predictor of the presence of LV LGE in SLE patients, which represents fibrosis/necrosis and potentially less reversible myocardial injury. The impaired LV GLS represents the longitudinal fibrous systolic dysfunction located predominantly in the sub-endocardium (10,31), confirming past reports of early SLE being prone to endocardial microvascular injury and diffuse myocarditis (32,33). Accordingly, CMR-derived LV strain analysis may have an additive value to some extent when SLE patients cannot undergo enhanced CMR scans, such as in patients with lupus nephritis.

In our cohort, biventricular interaction was observed in SLE-related cardiac involvement. The RVEF had a good correlation with LV global strain and diastolic strain rate, whereas LVEF had relatively weak or no correlation with $\mathrm{RV}$ strain parameters. The interdependence between the 
Table 5 Univariate and multivariate linear regression analysis to identify association between strain parameters and other clinical variables

\begin{tabular}{|c|c|c|c|c|c|c|c|c|c|c|}
\hline \multirow{3}{*}{ Parameters } & \multicolumn{10}{|c|}{$\operatorname{LV}(\beta)$} \\
\hline & \multicolumn{2}{|c|}{ GRS } & \multicolumn{2}{|c|}{ GCS } & \multicolumn{2}{|c|}{ GLS } & \multicolumn{2}{|c|}{ PDGRSR } & \multicolumn{2}{|c|}{ PDGCSR } \\
\hline & Univariate & Multivariate & Univariate & Multivariate & Univariate & Multivariate & Univariate & Multivariate & Univariate & Multivariate \\
\hline \multicolumn{11}{|l|}{ LVEF } \\
\hline RVEF & $0.526^{*}$ & $0.669^{*}$ & $-0.615^{\star}$ & $-0.691^{*}$ & $-0.754^{\star}$ & $-0.715^{\star}$ & $-0.483^{*}$ & $-0.536^{\star}$ & $0.406^{*}$ & $0.406^{*}$ \\
\hline Pericardial effusion & 0.354 & 0.226 & -0.041 & - & 0.133 & - & -0.163 & -0.250 & 0.023 & - \\
\hline $\mathrm{PAH}$ & -0.086 & - & 0.228 & - & 0.248 & - & -0.032 & -0.060 & -0.229 & - \\
\hline Anti-RNP & 0.071 & 0.257 & 0.036 & -0.212 & 0.161 & - & 0.056 & - & -0.041 & - \\
\hline
\end{tabular}

Table 5 (continued)

\begin{tabular}{|c|c|c|c|c|c|c|c|c|c|c|}
\hline \multirow{2}{*}{ Parameters } & \multicolumn{2}{|c|}{$\begin{array}{c}\text { LV }(\beta) \\
\text { PDGLSR }\end{array}$} & \multicolumn{8}{|c|}{$\mathrm{RV}(\beta)$} \\
\hline & Univariate & Multivariate & Univariate & Multivariate & Univariate & Multivariate & Univariate & Multivariate & Univariate & Multivariate \\
\hline LVEF & & & $-0.298^{\star}$ & -0.245 & $-0.325^{\star}$ & -0.240 & $0.382^{*}$ & $0.371^{*}$ & 0.198 & - \\
\hline RVEF & $0.499^{*}$ & $0.461^{*}$ & & & & & & & & \\
\hline Pericardial effusion & -0.042 & - & 0.113 & - & 0.262 & - & $-0.299^{\star}$ & $-0.285^{*}$ & -0.183 & - \\
\hline $\mathrm{PAH}$ & 0.014 & - & 0.185 & - & $0.383^{*}$ & $0.319^{*}$ & -0.133 & - & -0.008 & - \\
\hline Anti-RNP & -0.092 & - & $0.395^{\star}$ & $0.359^{*}$ & $0.315^{*}$ & - & -0.260 & - & 0.096 & - \\
\hline
\end{tabular}

${ }^{*} \mathrm{P}<0.05$. The $\beta$ indicates normalized $\beta$ values. LV, left ventricle; RV, right ventricle; EF, ejection fraction; GRS, global radial peak strain; GCS, global circumferential peak strain; GLS, global longitudinal peak strain; PSGRSR, peak systolic GRS rate; PSGCSR, peak systolic GCS rate; PSGLSR, peak systolic GLS rate; PDGRSR, peak diastolic GRS rate; PDGCSR, peak diastolic GCS rate; PDGLSR, peak diastolic GLS rate.

left and right ventricles can be explained appropriately by 3D echocardiography investigation (34): circumferentiallyoriented myofibers located on the RV epicardial surface encompass the sub-pulmonary infundibulum and advance more or less parallelly to the atrioventricular groove; a primary mechanism of $\mathrm{RV}$ pump function is bulging of the interventricular septum into the RV during $\mathrm{LV}$ contraction and stretching of the free wall over the septum, causing RV shortening in the anteroposterior direction. Additionally, LGE in the interventricular septum and RV insertion points might facilitate biventricular interaction, which is consistent with the research findings of $\mathrm{Wu}$ et al. (35) and Puntmann et al. (36). Studies of pulmonary hypertension have also reported that the extent of LGE in the RV insertion points was correlated with RV functional parameters (RV volumes, mass, EF, and longitudinal strain) $(37,38)$.

Functional parameters of RV, including EF and myocardial strain, were impaired before the LV. Guo et al. (14) also found that the RV was affected first in cardiac impairment after exclusion of SLE patients with CAD. Several studies have proposed that the cause of RV dysfunction might be related to $\mathrm{PAH}$, the involvement of smaller-sized and medium-sized arteries, or microvascular injury related to endothelial dysfunction induced by antiphospholipid antibodies (APL) $(14,35,39)$. Furthermore, we found that RV GLS was positively correlated with the PAH. The SLE group with preserved RVEF had a lower RV GCS and PDGLSR than normal controls. In patients with $\mathrm{PAH}, \mathrm{RV}$ longitudinal strain obtained by CMR tagging and CMR-based heart deformation analysis was also positively correlated with mean pulmonary artery pressure and pulmonary vascular resistance $(38,40)$. These findings were consistent with the results of $\mathrm{Wu}$ et al. (35) and further confirmed that the RV contraction function 

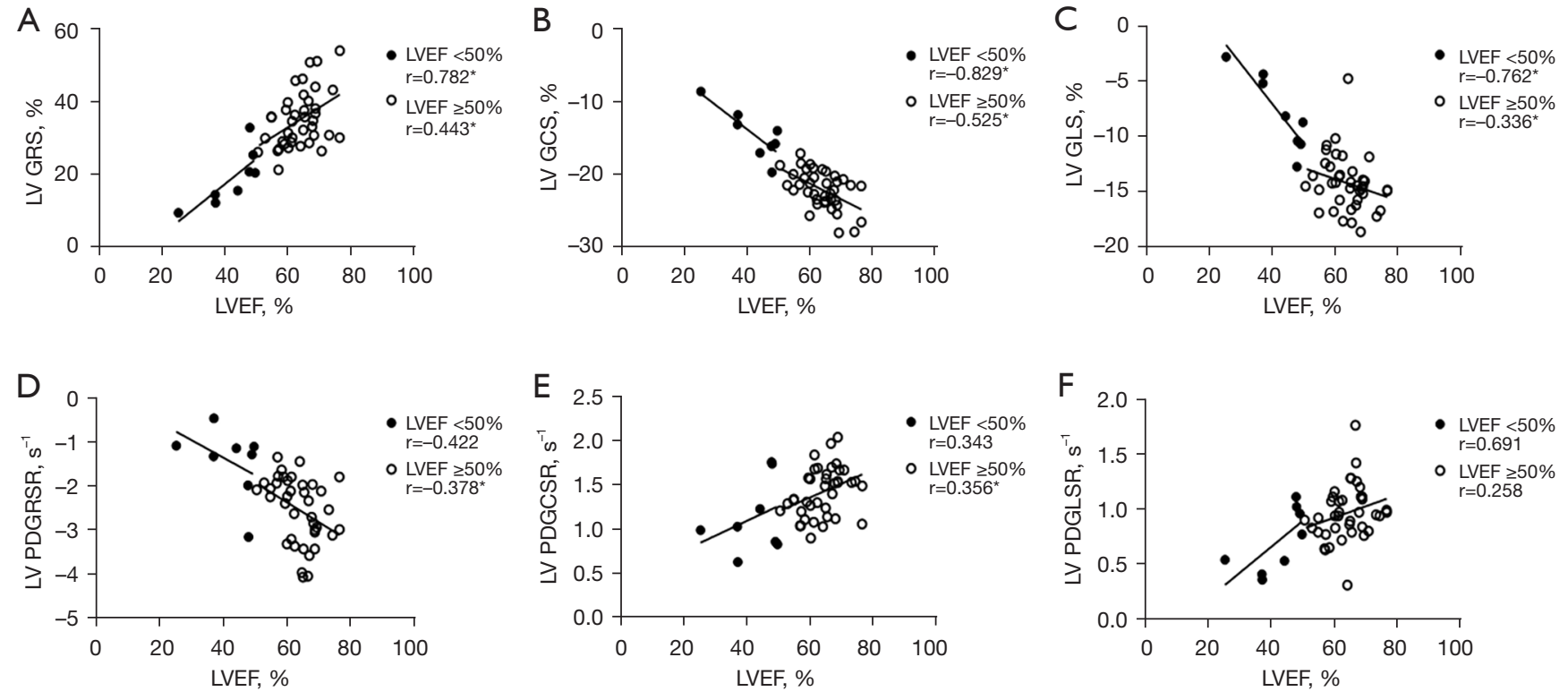

Figure 5 Correlation analysis of LVEF. The correlation results between LVEF and LV GRS (A), GCS (B), GLS (C), and PDGRSR (D), PDGCSR (E), and PDGLSR (F) in patients with SLE. * $\mathrm{P}<0.05$. LVEF, left ventricular ejection fraction; LV, left ventricular; SLE, systemic lupus erythematosus; GRS, global radial peak strain; GCS, global circumferential peak strain; GLS, global longitudinal peak strain; PDGRSR, peak diastolic GRS rate; PDGCSR, peak diastolic GCS rate; PDGLSR, peak diastolic GLS rate; SLE, systemic lupus erythematous.
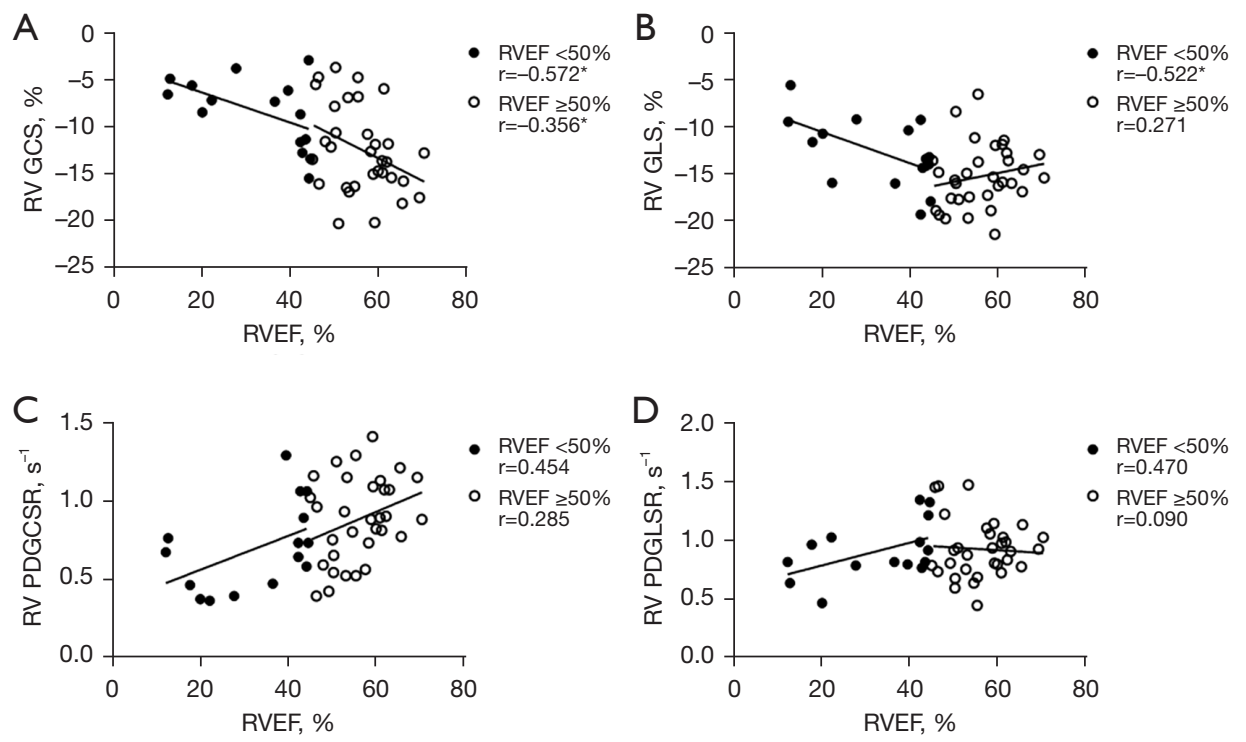

Figure 6 Correlation analysis of RVEF. The correlation results between RVEF and RV GCS (A), GLS (B), PDGCSR (C), and PDGLSR (D) in patients with SLE. *P<0.05. RVEF, right ventricular ejection fraction; RV, right ventricular; GCS, global circumferential peak strain; SLE, systemic lupus erythematous. GLS, global longitudinal peak strain; PDGCSR, peak diastolic GCS rate; PDGLSR, peak diastolic GLS rate 


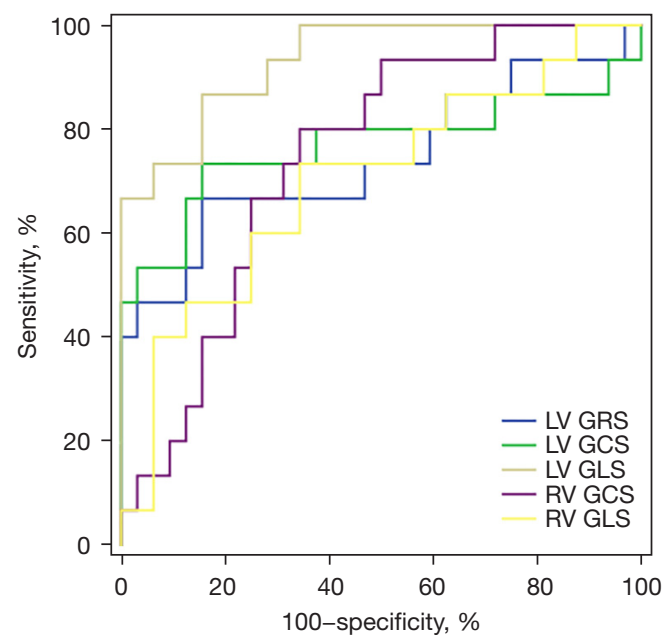

Figure 7 ROC curves of LV and RV global peak strain to predict RV dysfunction (RV ejection fraction <45\%) in patients with SLE. Among these strain parameters, LV GLS, with a cut-off value of $-13.38 \%$, has a significantly higher discriminatory power for RV dysfunction (sensitivity $86.70 \%$; specificity $84.38 \%$ ). ROC, receiver operating characteristic; RV, right ventricular; LV, left ventricular; SLE, systemic lupus erythematous; GLS, global longitudinal peak strain.

Table 6 Inter- and intra-observer variability of CMR tissue tracking

\begin{tabular}{|c|c|c|c|c|}
\hline \multirow{2}{*}{ Parameters } & \multicolumn{2}{|c|}{ Intraobserver $(\mathrm{n}=25)$} & \multicolumn{2}{|c|}{ Interobserver $(n=25)$} \\
\hline & ICC & $95 \% \mathrm{Cl}$ & ICC & $95 \% \mathrm{Cl}$ \\
\hline \multicolumn{5}{|l|}{ LV } \\
\hline GRS & 0.931 & $0.852-0.969$ & 0.931 & $0.854-0.969$ \\
\hline GCS & 0.957 & $0.905-0.981$ & 0.939 & $0.869-0.972$ \\
\hline PSGCSR & 0.814 & $0.625-0.913$ & 0.833 & $0.664-0.921$ \\
\hline PSGLSR & 0.683 & $0.407-0.846$ & 0.811 & $0.622-0.911$ \\
\hline PDGRSR & 0.813 & $0.619-0.913$ & 0.771 & $0.550-0.890$ \\
\hline GCS & 0.945 & $0.880-0.975$ & 0.891 & $0.772-0.950$ \\
\hline GLS & 0.875 & $0.739-0.943$ & 0.836 & $0.664-0.923$ \\
\hline PSGCSR & 0.651 & $0.358-0.829$ & 0.729 & $0.480-0.869$ \\
\hline PSGLSR & 0.698 & $0.422-0.855$ & 0.633 & $0.339-0.816$ \\
\hline PDGCSR & 0.700 & $0.399-0.860$ & 0.629 & $0.332-0.813$ \\
\hline PDGLSR & 0.622 & $0.314-0.813$ & 0.627 & $0.331-0.812$ \\
\hline
\end{tabular}

CMR, cardiovascular magnetic resonance; ICC, intraclass correlation coefficient; CI, confidence interval; LV, left ventricle; RV, right ventricle; GRS, global radial peak strain; GCS, global circumferential peak strain; GLS, global longitudinal peak strain; PSGRSR, peak systolic GRS rate; PSGCSR, peak systolic GCS rate; PSGLSR, peak systolic GLS rate; PDGRSR, peak diastolic GRS rate; PDGCSR, peak diastolic GCS rate; PDGLSR, peak diastolic GLS rate. 
in the longitudinal direction was gradually impaired due to PAH before that in the circumferential direction, as RV PDGLSR was significantly lower in our SLE participants with preserved RVEF and may have been more sensitive than RV GLS. Later, the continuously increasing RV afterload caused by PAH engendered RV adaptive myocardial hypertrophy and changed RV myocardial fibers into a more circumferential direction, resulting in a decrease of RV GCS and RVEF. Currently, the RV longitudinal strain driven by the deep RV myocardium with longitudinal arrangements is recommended to assess RV function in patients with suspected RV dysfunction, such as congenital abnormalities, arrhythmogenic RV cardiomyopathy, and PAH $(10,13,41)$. However, Kind et al. (42) reported on the circumferential compression of the RV chamber or movement of the free wall toward the septum (bellows action), which seem to be a better predictor of RVEF than longitudinal shortening in PAH. This difference may be related to different deformation analysis technologies or the heterogeneity of the patient population. These findings need to be verified in future studies with larger cohorts or multi-center results.

Among SLE-related autoimmune antibodies, only antiRNP antibody was independently correlated with RV GCS. As reported by Zawadowski et al. (43), elevated anti-RNP levels have been associated with lupus myocarditis in SLE. In addition, anti-RNP antibody has also been associated with interstitial lung disease, which may affect the RV afterload $(44,45)$. Furthermore, pericardial effusion was associated with RV PDGCSR, which might explain that the thin RV wall is easily affected by pericarditis. Hence, SLE patients with anti-RNP antibody positivity, PAH, and pericardial effusion detected by echocardiography could be considered to further CMR examination. However, these reproducibility results resemble the reported results in several articles $(23,41,46)$; the reproducibility for ventricular peak strain rate obtained by CVI42 software was relatively lower than the peak strain values, particularly for RV in this study. Therefore, there is limited value in the strain rate for the exact quantification and determination of RV function.

The myocardial strain changes in SLE appear to precede overt RVEF impairment. Interestingly, ROC curve analysis showed that LV GLS with an optimal cut-off value of $-13.38 \%$ had the most powerful diagnostic value for RV dysfunction, compared with the remaining biventricular global peak strain indices with moderate diagnostic power. We hypothesized that the impairment of LV endocardial longitudinal fibers might be the most susceptible and significant in SLE-related cardiac involvement. In addition to RV involvement caused by microvasculitis, myocarditis, and pulmonary hypertension in patients with $\operatorname{SLE}(14,35,39)$, biventricular interaction may lead to RV dysfunction when LV dysfunction is present (34). Furthermore, LV GLS was correlated with LVEF and LGE in this study. Hence, more attention should be paid to LV GLS in clinical decision-making and prognostication.

There were some limitations in this study. First, this was a single-center study with small sample size, and the verification effect was therefore relatively limited. Second, CMR tissue tracking lacks a reference standard, and its accuracy needs to be further verified. Improvements to the tissue-tracking algorithm of post-processing software are needed to improve the reproducibility of RV strain and biventricular strain rate analysis. Third, most patients with SLE were initially diagnosed in our study, and the systolic strain rates' impairment was therefore not obvious. The clinical significance of the systolic strain rate in SLE needs to be studied further. Finally, follow-up observations were not conducted to verify whether immunosuppressive and anti-inflammatory treatment could affect myocardial deformation and function.

To summarize, biventricular strain parameters detected by CMR tissue tracking are sensitive markers of subclinical ventricular functional impairment before EF reduction at an early stage in SLE patients. The EF, LGE, PAH, anti-body RNP positive, and pericardial effusion are associated with the myocardial strain parameters. A possible recommendation to be made is for biventricular strain analysis to be included in early cardiac functional assessment in SLE patients (particularly LV GLS), which might assist in clinical decision-making and disease monitoring.

\section{Acknowledgments}

Funding: This work was supported by the National Natural Science Foundation of China (81601462); the Key Research \& Development Project of Science and Technology of Sichuan Province (2021YFS0142); and the 1.3.5 Project for Disciplines of Excellence, West China Hospital, Sichuan University (ZYGD1801).

\section{Footnote}

Reporting Checklist: The authors have completed the STARD reporting checklist. Available at https://dx.doi. org/10.21037/qims-21-520 
Conflicts of Interest: All authors have completed the ICMJE uniform disclosure form (available at https://dx.doi. org/10.21037/qims-21-520). The authors have no conflicts of interest to declare.

Ethical Statement: The authors are accountable for all aspects of the work in ensuring that questions related to the accuracy or integrity of any part of the work are appropriately investigated and resolved. The study was conducted in accordance with the Declaration of Helsinki (as revised in 2013). The study was approved by institutional ethics review board of West China Hospital and informed consent was provided by all individual participants.

Open Access Statement: This is an Open Access article distributed in accordance with the Creative Commons Attribution-NonCommercial-NoDerivs 4.0 International License (CC BY-NC-ND 4.0), which permits the noncommercial replication and distribution of the article with the strict proviso that no changes or edits are made and the original work is properly cited (including links to both the formal publication through the relevant DOI and the license). See: https://creativecommons.org/licenses/by-nc-nd/4.0/.

\section{References}

1. Griffith GC, Vural IL. Acute and subacute disseminated lupus erythematosus; a correlation of clinical and postmortem findings in eighteen cases. Circulation 1951;3:492-500.

2. Miner JJ, Kim AH. Cardiac manifestations of systemic lupus erythematosus. Rheum Dis Clin North Am 2014;40:51-60.

3. Jain D, Halushka MK. Cardiac pathology of systemic lupus erythematosus. J Clin Pathol 2009;62:584-92.

4. Doria A, Iaccarino L, Sarzi-Puttini P, Atzeni F, Turriel M, Petri M. Cardiac involvement in systemic lupus erythematosus. Lupus 2005;14:683-6.

5. Bidani AK, Roberts JL, Schwartz MM, Lewis EJ. Immunopathology of cardiac lesions in fatal systemic lupus erythematosus. Am J Med 1980;69:849-58.

6. Fors Nieves CE, Izmirly PM. Mortality in Systemic Lupus Erythematosus: an Updated Review. Curr Rheumatol Rep 2016;18:21.

7. Mavrogeni S, Karabela G, Stavropoulos E, Plastiras S, Spiliotis G, Gialafos E, Kolovou G, Sfikakis PP, Kitas GD. Heart failure imaging patterns in systemic lupus erythematosus. Evaluation using cardiovascular magnetic resonance. Int J Cardiol 2014;176:559-61.

8. Apte M, McGwin G Jr, Vilá LM, Kaslow RA, Alarcón GS, Reveille JD; LUMINA Study Group. Associated factors and impact of myocarditis in patients with SLE from LUMINA, a multiethnic US cohort (LV). corrected. Rheumatology (Oxford) 2008;47:362-7.

9. Scatteia A, Baritussio A, Bucciarelli-Ducci C. Strain imaging using cardiac magnetic resonance. Heart Fail Rev 2017;22:465-76.

10. Claus P, Omar AMS, Pedrizzetti G, Sengupta PP, Nagel E. Tissue Tracking Technology for Assessing Cardiac Mechanics: Principles, Normal Values, and Clinical Applications. JACC Cardiovasc Imaging 2015;8:1444-60.

11. Hor KN, Baumann R, Pedrizzetti G, Tonti G, Gottliebson WM, Taylor M, Benson DW, Mazur W. Magnetic resonance derived myocardial strain assessment using feature tracking. J Vis Exp 2011;(48):2356.

12. Hu BY, Wang J, Yang ZG, Ren Y, Jiang L, Xie LJ, Liu X, Gao Y, Shen MT, Xu HY, Shi K, Li ZL, Xia CC, Peng WL, Deng MY, Li H, Guo YK. Cardiac magnetic resonance feature tracking for quantifying right ventricular deformation in type 2 diabetes mellitus patients. Sci Rep 2019;9:11148

13. Liu H, Fu H, Guo YK, Yang ZG, Xu HY, Shuai X, Xu R, Li ZL, Xia CC, He Y, Zhou XY. The prognostic value of right ventricular deformation derived from cardiac magnetic resonance tissue tracking for all-cause mortality in light-chain amyloidosis patients. Cardiovasc Diagn Ther 2020;10:161-72.

14. Guo Q, Wu LM, Wang Z, Shen JY, Su X, Wang CQ, Gong XR, Yan QR, He Q, Zhang W, Xu JR, Jiang M, Pu J. Early Detection of Silent Myocardial Impairment in Drug-Naive Patients With New-Onset Systemic Lupus Erythematosus: A Three-Center Prospective Study. Arthritis Rheumatol 2018;70:2014-24.

15. Wu R, An DA, Hu J, Jiang M, Guo Q, Xu JR, Wu LM. The apparent diffusion coefficient is strongly correlated with extracellular volume, a measure of myocardial fibrosis, and subclinical cardiomyopathy in patients with systemic lupus erythematosus. Acta Radiol 2018;59:287-95.

16. Azevedo CF, Amado LC, Kraitchman DL, Gerber BL, Osman NF, Rochitte CE, Edvardsen T, Lima JA. Persistent diastolic dysfunction despite complete systolic functional recovery after reperfused acute myocardial infarction demonstrated by tagged magnetic resonance imaging. Eur Heart J 2004;25:1419-27.

17. Hochberg MC. Updating the American College of Rheumatology revised criteria for the classification 
of systemic lupus erythematosus. Arthritis Rheum 1997;40:1725.

18. Yee CS, Farewell VT, Isenberg DA, Griffiths B, Teh LS, Bruce IN, Ahmad Y, Rahman A, Prabu A, Akil M, McHugh N, Edwards C, D'Cruz D, Khamashta MA, Gordon C. The use of Systemic Lupus Erythematosus Disease Activity Index-2000 to define active disease and minimal clinically meaningful change based on data from a large cohort of systemic lupus erythematosus patients. Rheumatology (Oxford) 2011;50:982-8.

19. Bistoquet A, Oshinski J, Skrinjar O. Myocardial deformation recovery from cine MRI using a nearly incompressible biventricular model. Med Image Anal 2008;12:69-85.

20. Le TT, Tan RS, De Deyn M, Goh EP, Han Y, Leong BR, Cook SA, Chin CW. Cardiovascular magnetic resonance reference ranges for the heart and aorta in Chinese at $3 \mathrm{~T}$. J Cardiovasc Magn Reson 2016;18:21.

21. Chan YH. Biostatistics 104: correlational analysis. Singapore Med J 2003;44:614-9.

22. Aurich M, Keller M, Greiner S, Steen H, Aus dem Siepen F, Riffel J, Katus HA, Buss SJ, Mereles D. Left ventricular mechanics assessed by two-dimensional echocardiography and cardiac magnetic resonance imaging: comparison of high-resolution speckle tracking and feature tracking. Eur Heart J Cardiovasc Imaging 2016;17:1370-8.

23. Liu B, Dardeer AM, Moody WE, Hayer MK, Baig S, Price AM, Leyva F, Edwards NC, Steeds RP. Reference ranges for three-dimensional feature tracking cardiac magnetic resonance: comparison with two-dimensional methodology and relevance of age and gender. Int J Cardiovasc Imaging 2018;34:761-75.

24. Wang J, Shi K, Xu HY, Zhao Q, Liu X, Gao Y, Yu H, Guo YK, Yang ZG. Left Ventricular Deformation in Patients with Connective Tissue Disease: Evaluated by 3.0T Cardiac Magnetic Resonance Tissue Tracking. Sci Rep 2019;9:17913.

25. Li X, Shi K, Yang ZG, Guo YK, Huang S, Xia CC, He S, Li ZL, Li C, He Y. Assessing right ventricular deformation in hypertrophic cardiomyopathy patients with preserved right ventricular ejection fraction: a 3.0-T cardiovascular magnetic resonance study. Sci Rep 2020;10:1967.

26. Luo T, Wang Z, Chen Z, Yu E, Fang C. Layer-specific strain and dyssynchrony index alteration in new-onset systemic lupus erythematosus patients without cardiac symptoms. Quant Imaging Med Surg 2021;11:1271-83.

27. Smiseth OA, Torp H, Opdahl A, Haugaa KH, Urheim S. Myocardial strain imaging: how useful is it in clinical decision making? Eur Heart J 2016;37:1196-207.

28. Hong S, Son J, Kim B, Kim B, Lee Y, Lee J, Ryu J, Choi J, Chang S, Kim K. Usefulness of Echocardiography in Early Detection of Global and Regional Left Ventricular Diastolic Dysfunction in Systemic Lupus Erythematosus. J Am Coll Cardiol 2012;59:E1250.

29. Leone P, Cicco S, Prete M, Solimando AG, Susca N, Crudele L, Buonavoglia A, Colonna P, Dammacco F, Vacca A, Racanelli V. Early echocardiographic detection of left ventricular diastolic dysfunction in patients with systemic lupus erythematosus asymptomatic for cardiovascular disease. Clin Exp Med 2020;20:11-9.

30. du Toit R, Herbst PG, Ackerman C, Pecoraro AJ, du Toit RH, Hassan K, Joubert LH, Reuter H, Doubell AF. Myocardial injury in systemic lupus erythematosus according to cardiac magnetic resonance tissue characterization: clinical and echocardiographic features. Lupus 2020;29:1461-8.

31. Kocica MJ, Corno AF, Carreras-Costa F, Ballester-Rodes M, Moghbel MC, Cueva CN, Lackovic V, Kanjuh VI, Torrent-Guasp F. The helical ventricular myocardial band: global, three-dimensional, functional architecture of the ventricular myocardium. Eur J Cardiothorac Surg 2006;29 Suppl 1:S21-40.

32. Goykhman P, Mehta PK, Minissian M, Thomson LE, Berman DS, Ishimori ML, Wallace DJ, Weisman MH, Shufelt CL, Bairey Merz CN. Subendocardial ischemia and myocarditis in systemic lupus erythematosus detected by cardiac magnetic resonance imaging. J Rheumatol 2012;39:448-50.

33. Mavrogeni S, Koutsogeorgopoulou L, MarkousisMavrogenis G, Bounas A, Tektonidou M, Lliossis SC, Daoussis D, Plastiras S, Karabela G, Stavropoulos E, Katsifis G, Vartela V, Kolovou G. Cardiovascular magnetic resonance detects silent heart disease missed by echocardiography in systemic lupus erythematosus. Lupus 2018;27:564-71.

34. Kovács A, Lakatos B, Tokodi M, Merkely B. Right ventricular mechanical pattern in health and disease: beyond longitudinal shortening. Heart Fail Rev 2019;24:511-20.

35. Wu R, Shi RY, An DAL, Chen BH, Jiang M, Bacyinski A, Han TT, Deen JM, Kaddurah H, Hu J, Xu JR, Wu LM. Biventricular tissue tracking demonstrating associations between left ventricular myocardial extracellular volume, pulmonary artery pressure, and reduced right ventricular ejection fraction in patients with systemic lupus erythematosus using cardiovascular MRI. Clin Radiol 
2020;75:237.e17-25.

36. Puntmann VO, D'Cruz D, Smith Z, Pastor A, Choong P, Voigt T, Carr-White G, Sangle S, Schaeffter T, Nagel E. Native myocardial T1 mapping by cardiovascular magnetic resonance imaging in subclinical cardiomyopathy in patients with systemic lupus erythematosus. Circ Cardiovasc Imaging 2013;6:295-301.

37. Sanz J, Dellegrottaglie S, Kariisa M, Sulica R, Poon M, O'Donnell TP, Mehta D, Fuster V, Rajagopalan S. Prevalence and correlates of septal delayed contrast enhancement in patients with pulmonary hypertension. Am J Cardiol 2007;100:731-5.

38. Tao X, Liu M, Liu W, Xie W, Wan J, Zhai Z, Wang C. $\mathrm{CMR}$-based heart deformation analysis for quantification of hemodynamics and right ventricular dysfunction in patients with CTEPH. Clin Respir J 2020;14:277-84.

39. Mineo C. Inhibition of nitric oxide and antiphospholipid antibody-mediated thrombosis. Curr Rheumatol Rep 2013;15:324.

40. Shehata ML, Harouni AA, Skrok J, Basha TA, Boyce D, Lechtzin N, Mathai SC, Girgis R, Osman NF, Lima JA, Bluemke DA, Hassoun PM, Vogel-Claussen J. Regional and global biventricular function in pulmonary arterial hypertension: a cardiac MR imaging study. Radiology 2013;266:114-22.

41. Liu X, Yang ZG, Gao Y, Xie LJ, Jiang L, Hu BY, Diao KY,
Shi K, Xu HY, Shen MT, Ren Y, Guo YK. Left ventricular subclinical myocardial dysfunction in uncomplicated type 2 diabetes mellitus is associated with impaired myocardial perfusion: a contrast-enhanced cardiovascular magnetic resonance study. Cardiovasc Diabetol 2018;17:139.

42. Kind T, Mauritz GJ, Marcus JT, van de Veerdonk M, Westerhof N, Vonk-Noordegraaf A. Right ventricular ejection fraction is better reflected by transverse rather than longitudinal wall motion in pulmonary hypertension. J Cardiovasc Magn Reson 2010;12:35.

43. Zawadowski GM, Klarich KW, Moder KG, Edwards WD, Cooper LT Jr. A contemporary case series of lupus myocarditis. Lupus 2012;21:1378-84.

44. Dima A, Jurcut C, Baicus C. The impact of anti-U1-RNP positivity: systemic lupus erythematosus versus mixed connective tissue disease. Rheumatol Int 2018;38:1169-78.

45. Lhote R, Grenier P, Haroche J, Miyara M, Boussouar S, Mathian A, Pha M, Amoura Z, Cohen Aubart F. Characterization of Interstitial Lung Disease Associated With Anti-Ribonucleoprotein Antibodies. J Clin Rheumatol 2020;26:327-33.

46. Qu YY, Paul J, Li H, Ma GS, Buckert D, Rasche V. Left ventricular myocardial strain quantification with two- and three-dimensional cardiovascular magnetic resonance based tissue tracking. Quant Imaging Med Surg 2021;11:1421-36.

Cite this article as: $\mathrm{Pu} \mathrm{H}$, Cui $\mathrm{B}$, Liu $\mathrm{J}, \mathrm{He} \mathrm{W}$, Zhou $\mathrm{X}$, Lin H, Peng L. Characterization and clinical significance of biventricular mechanics in patients with systemic lupus erythematosus by $3 \mathrm{~T}$ cardiovascular magnetic resonance tissue tracking. Quant Imaging Med Surg 2022;12(2):1079-1697. doi: 10.21037/qims-21-520 